\title{
ON TIME-VARYING BIT-ALLOCATION MAINTAINING INPUT-OUTPUT STABILITY: A CONVEX PARAMETERIZATION
}

\author{
Sridevi V. Sarma, Munther A. Dahleh, Srinivasa Salapaka* \\ Massachusetts Institute of Technology \\ Laboratory of Information and Decision Systems \\ sree@mit.edu, dahleh@mit.edu, salapaka@uiuc.edu
}

\begin{abstract}
In [9], we constructed a parameterization of general time-varying quantizers. The construction is general in that it can have infinite memory and be time-varying in that the strategy it follows in allocating a total of $R$ bits to its inputs, is a function of time. We derived sufficient conditions for input-output stability as functions of the quantizer's time-dependent bit-allocation strategy for bounded reference inputs. Our generalized construction of the quantizer also led to the result that the set of allocation strategies that maintains stability for bounded inputs is convex, allowing the search for the most efficient strategy to ensure stability to be formulated as a convex optimization problem. In this paper, we extend our stability analysis and derive sufficient conditions for decaying reference signals. We further show that the set of allocation strategies that maintains stability remains convex, as in the case for bounded inputs. We then compute optimal bit-allocation strategies for a class of finite-memory quantizers for various plant and controller pairs, and observe that the most efficient strategies are non-trivial and time-varying. Throughout, we consider a system in which the plant and feedback controller are separated by a noiseless finiterate communication channel.
\end{abstract}

\section{INTRODUCTION}

Due to the information-rich world we live in today, control in distributed, asynchronous, networked environments is in demand. Communication links that have rate limitations, delays, and that are noisy, are now heavily connected to control systems, and the interactions between the two cannot be ignored. We consider the control system, shown in Figure 1, in which a discrete-time plant and feedback controller are separated by a finite-rate noiseless communication channel.

Most research in this area study the case in which no external inputs are applied to the control system, and derive conditions on the channel rate required for various notions of asymptotic stability [1, 2, 3, 4, 5, 6, 7, 8, 10, 11]. When $r=0$, assuming a known $G$ and a stabilizing $K$, the only parameter characterizing all the signals in the closed-loop system is the initial condition of the plant, $x_{0}$. Therefore, the channel encoder, $E$, and decoder, $D$ can spend their efforts transmitting more and more information about $x_{0}$ through the channel. This amounts to allocating more and more bits over time to $x_{0}$. Ultimately, the state trajectory

\footnotetext{
*This research has been supported by AFOSR: 6892167.
}

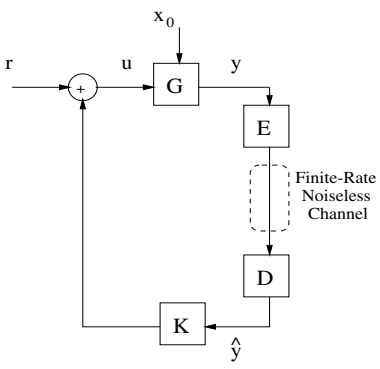

Fig. 1. General Control System

can be computed exactly by the decoder, which enables various forms of asymptotic stability to be achieved, since $K$ is assumed to be stabilizing.

If $r$ is a non-zero input, the number of parameters that characterize the closed-loop dynamics can be infinite $\left(x_{0}\right.$ plus $r_{k}$ for $k \geq 0$ ), and hence the approximations of these parameters by the decoder do not asymptotically converge to their actual values. Statements about what happens to the system's state vector cannot, in general, be made. The best one can do is to allow the approximations of these parameters (or functions of these parameters such as the output values $y_{k}$ for $k \geq 0$ ) to improve over time by allocating more and more bits to them, and analyze input-output stability.

In this paper, we consider reference inputs from two parameterized classes of signals, $\mathcal{C}_{r}$, and derive corresponding conditions on the channel rate required for input-output stability. We define the closed-loop system to be input-output stable if for all $r \in \mathcal{C}_{r}$, there exists a $\mu<\infty$ and $\alpha<\infty$ such that $\|y\|_{\infty} \leq \mu\|r\|_{\infty}+\alpha$.

\section{PROBLEM FORMULATION}

We assume $G$ to be a causal discrete-time LTI system with zero initial conditions ${ }^{1}$, and set the channel encoder to be a fixed-rate quantizer, $E=Q$, which has infinite memory, and is time-varying in that the strategy it follows in allocating a total of $R$ bits to all of the inputs up to time $t$, is a function of $t$. We assume that the channel can transmit $R$ bits instantaneously with each use. The channel decoder, $D$, receives more information on the values of $y$ and sends these

\footnotetext{
${ }^{1}$ Results in this paper hold even with nonzero initial conditions.
} 
updates to the controller. $K$ is a causal discrete-time LTI system that stabilizes $G$ in the ideal case of perfect communication. We investigate closed-loop input-output stability for each of the following classes of reference inputs, $\mathcal{C}_{r}$.

1. Bounded Signals: $\mathcal{C}_{r}=l_{\infty, \bar{r}}$, where $l_{\infty, \bar{r}}$ is the class of all signals that are bounded in magnitude by $\bar{r}$.

2. Decaying Signals: $\mathcal{C}_{r}=\mathcal{C}_{\gamma, \bar{r}}$, where $\mathcal{C}_{\gamma, \bar{r}}$ is the class of all signals that are bounded by the decaying function $\bar{r} \gamma^{k}$ for all $k \geq 0$ and $0<\gamma<1$, i.e., if $r \in \mathcal{C}_{\gamma, \bar{r}}$, then $\left|r_{k}\right| \leq \bar{r} \gamma^{k}$ for all $k \geq 0$.

\subsection{Limited-Rate Time-Varying $(\mathcal{R}, M)$-Quantizers}

Before stating the problems that we are interested in solving, we first define and model the general parameterized class of time-varying infinite memory $(\mathcal{R}, M)$-quantizers, first introduced in [9].

We view the quantizer as a module that approximates its input, which in general requires an infinite number of bits, with a finite number of bits. Formally speaking, an $(\mathcal{R}, M)$-quantizer with bit-rate $R$, is a sequence of causal time-varying operators, parameterized by an infinite dimensional positive-definite diagonal scale matrix, $M$, and an infinite dimensional rate matrix, $\mathcal{R}$, which, in general looks like

$$
\left[\begin{array}{ccccc}
R_{00} & 0 & 0 & \ldots & \cdots \\
R_{01} & R_{11} & 0 & 0 & \ldots \\
R_{02} & R_{12} & R_{22} & 0 & \cdots \\
\vdots & \vdots & \vdots & \ddots & \ddots
\end{array}\right]
$$

such that $\sum_{j} R_{i j}=R \forall i$.

The $(\mathcal{R}, M)$-quantizer saturates to output $M_{k k}$ when its input, $y(k)$, has magnitude greater than or equal to $M_{k k}$, i.e., when $|y(k)| \geq M_{k k}$. However, we denote the quantizer "valid" only when $|y(k)| \leq M_{k k}$, and define what the quantizer does in this case below.

Let $\hat{y}(i, j)$ be the quantized estimate of $y(i)$ at time $j$. Then, $\mathcal{R}$ determines that at time $t=0, R_{00}$ bits are used to quantize $y(0)$ to produce $\hat{y}(0,0)$; at time $t=1$, an additional $R_{01}$ bits are used to quantize $y(0)$ to produce $\hat{y}(0,1)$ and $R_{11}$ bits are used to quantize $y(1)$ to produce $\hat{y}(1,1)$, and so on. The accuracy of $\hat{y}(i, j)$ is within $\pm M_{i i} 2^{-\sum_{k=i}^{j} R_{i k}}$ of $y(i)$ for all $i \geq 0$.

More concretely, at any time $t$, the quantizer can be broken down into the five steps shown in Figure 2 when $|y(k)| \leq M_{k k}$ for $0 \leq k \leq t$.

First, the $i$ th component of the vector $\mathbf{y}^{t}$ is scaled by $\frac{1}{M_{i i}}$ for $i=0,1, \ldots, t$, to produce $\mathbf{z}^{t}$, where

$$
M_{t}=\left[\begin{array}{llll}
M_{00} & & & \\
& M_{11} & & \\
& & \ddots & \\
& & & M_{t t}
\end{array}\right] .
$$

The scaling by $M_{t}^{-1}$ ensures that $\left\|\mathbf{z}^{t}\right\|_{\infty} \leq 1$. Then, each element of $\mathbf{z}^{t}$ is converted into its binary representation, i.e., a string of ' 0 's and ' 1 's in the Decimal-to-Binary

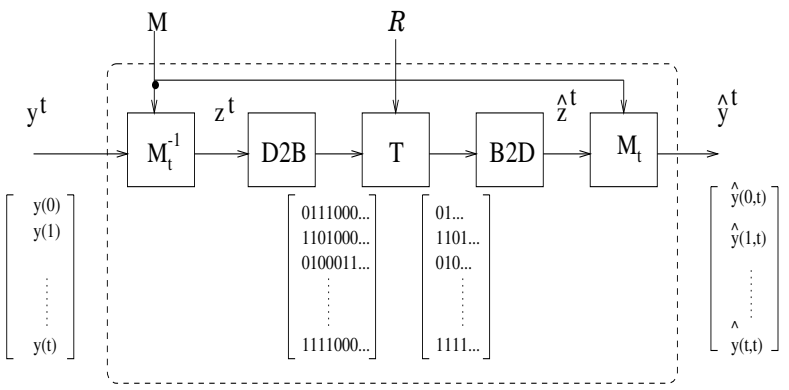

Fig. 2. Quantizer Operator at Time $t$

(D2B) converter. Next, each binary string is truncated according to the bit allocation strategy induced by $\mathcal{R}$. Specifically, the binary string representing $\mathbf{z}^{t}(i)=\frac{y(i)}{M_{i i}}$ is truncated to contain only its first $\sum_{j=i}^{t} R_{i j}$ bits. Note that this truncation induces an error of at most $2^{-\sum_{j=i}^{t} R_{i j}}$ in magnitude for $\mathbf{z}^{t}(i)$, i.e., $\left|\mathbf{z}^{t}(i)-\hat{\mathbf{z}}^{t}(i)\right| \leq 2^{-\sum_{j=i}^{t} R_{i j}}$.

As shown in Figure 2, the truncated binary string is converted back into its decimal representation, via the Binaryto-Decimal module (B2D), to produce $\hat{\mathbf{z}}^{t}$. Finally, $\hat{\mathbf{z}}^{t}$ is scaled by $M_{t}$ to produce $\hat{\mathbf{y}}^{t}$.

An upper bound on the error between each input component and its approximate output is:

$$
|y(k)-\hat{y}(k, t)| \leq M_{k k} 2^{-\sum_{i=k}^{t} R_{k i}}
$$

$\forall k \leq t$. Stated differently, if $|y(k)| \leq M_{k k}$ for $0 \leq k \leq t$, then there exists a $w(k, t)$ with $|w(k, t)| \leq 1$, such that

$$
\hat{y}(k, t)=y(k)+M_{k k} 2^{-\sum_{i=k}^{t} R_{k i}} w(k, t),
$$

for all $k \leq t$.

Note that the quantizer output above consists of only the estimates of $\mathbf{y}^{t}$ at time $t$. For analysis, we augment the output of the quantizer at time $t$ to be the vector of all estimates of $\mathbf{y}^{t}$ from time 0 to time $t$. We denote the augmented vector as $\hat{\mathbf{y}}_{a}^{t}$ as shown below.

We can then model the quantizer in its "valid" region as the following sequence of time-varying operators:

$$
\begin{gathered}
Q(\mathcal{R}, M)=\left\{Q_{t}: \mathbb{R}^{t+1} \rightarrow \mathbb{R}^{\frac{t(t+1)}{2}} \mid Q_{t}\left(\mathbf{y}^{t}\right)=\hat{\mathbf{y}}_{a}^{t}=\right. \\
\left.I_{t} \mathbf{y}^{t}+L_{t} \mathbf{w}_{a}^{t}, t \geq 0\right\},
\end{gathered}
$$

where

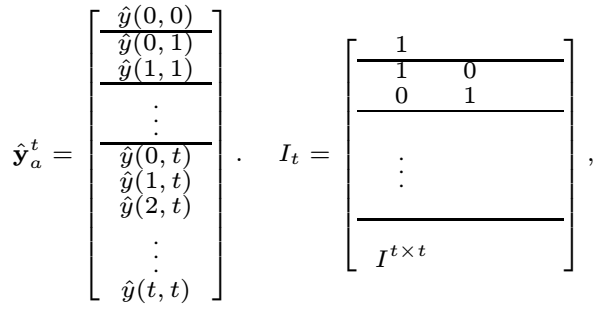




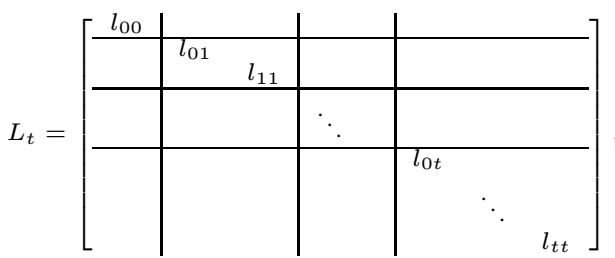

$$
\mathbf{w}_{a}^{t}=\left[\begin{array}{c}
w(0,0) \\
\hline w(0,1) \\
w(1,1) \\
\hline \vdots \\
\hline w(0, t) \\
w(1, t) \\
w(2, t) \\
\vdots \\
w(t, t)
\end{array}\right],
$$

with $l_{k s}=M_{k k} 2^{-\sum_{i=k}^{s} R_{k i}}$ for $s=0,1, \ldots, t$, and $k=0,1, \ldots, s$, and $\mathbf{w}_{a} \in l_{\infty}$ such that $\left\|\mathbf{w}_{a}\right\|_{\infty} \leq 1$. Note that the matrix parameters $\mathcal{R}$, and $M$ impact $Q_{t}\left(\mathbf{y}^{t}\right)$ through $L_{t}$ and $\mathbf{w}_{a}^{t}$.

\subsection{Channel Decoder}

The channel decoder's responsibility is to send the most accurate system output values that it can compute to the controller. In our analysis, this entails extracting the column vector $\hat{\mathbf{y}}^{t}$ from $\hat{\mathbf{y}}_{a}^{t}$, and is represented by the following $(t+1) \times \frac{t(t+1)}{2}$ matrix operator for any time $t$,

$$
D_{t}=\left[\begin{array}{l|l}
\quad \mathbf{0} & I_{(t+1) \times(t+1)}
\end{array}\right] .
$$

\subsection{Plant and Controller}

We represent the system $G$ and the controller $K$ as the following matrix multiplication operators at any time instance $t$ :

$$
\begin{aligned}
G_{t} & =\left[\begin{array}{ccccc}
G_{0} & & & & \\
G_{1} & G_{0} & & & \\
G_{2} & G_{1} & G_{0} & & \\
\vdots & & \ddots & \ddots & \\
G_{t} & \ldots & \ldots & G_{1} & G_{0}
\end{array}\right], \\
K_{t} & =\left[\begin{array}{ccccc}
K_{0} & & & & \\
K_{1} & K_{0} & & & \\
K_{2} & K_{1} & K_{0} & & \\
\vdots & & \ddots & \ddots & \\
K_{t} & K_{t-1} & \ldots & & K_{0}
\end{array}\right]
\end{aligned}
$$

If $G$ and $K$ are finite-dimensional, with state-space descriptions $\left(A_{g}, B_{g}, C_{g}, D_{g}\right)$ and $\left(A_{k}, B_{k}, C_{k}, D_{k}\right)$, respectively, then $G_{0}=D_{g}, G_{j}=C_{g} A_{g}^{j-1} B_{g}$ for $j \geq 1$, and $K_{0}=D_{k}$, and $K_{j}=C_{k} A_{k}^{j-1} B_{k}$ for $j \geq 1$.

Figure 3 illustrates the closed-loop system at time $t$ when the quantizer is modeled as an endogenous disturbance as described in section 2.1 , with

$$
\mathbf{r}^{t}=\left[\begin{array}{c}
r(0) \\
r(1) \\
\vdots \\
r(t)
\end{array}\right] \& \quad \mathbf{u}^{t}=\left[\begin{array}{c}
u(0) \\
u(1) \\
\vdots \\
u(t)
\end{array}\right]
$$

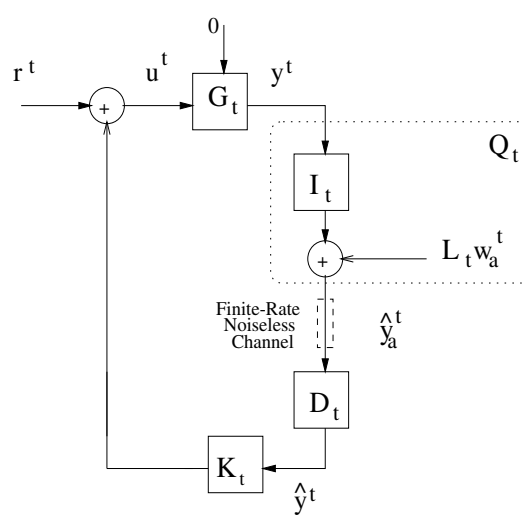

Fig. 3. Control System at Time $t$

\subsection{Problem Statement}

We are interested in solving the following problems for each class of reference inputs, $\mathcal{C}_{r}$, defined earlier.

1. Given $G, K, \mathcal{C}_{r}$, and a rate matrix, $\mathcal{R}$, find a set of scale matrices, $M$, that maintain input-output stability.

2. Given $G, K, \mathcal{C}_{r}$, characterize the set of all rate matrices, $\mathcal{R}$, such that the system is input-output stable.

3. Within the set of stabilizing rate matrices, find the minimum transmission rate, $R$, of the channel.

\section{BOUNDED MAGNITUDE REFERENCE SIGNALS}

In this section, we consider the case where $\mathcal{C}_{r}=l_{\infty, \bar{r}}$. Let $T_{1, t}$ be the operator from $\mathbf{r}^{t}$ to $\mathbf{y}^{t}$ at time $t$, and let $T_{2, t}$ be the operator from $\mathbf{w}_{a}^{t}$ to $\mathbf{y}^{t}$ at time $t$, i.e., $T_{1, t}=\left(I-G_{t} K_{t}\right)^{-1} G_{t}$ and $T_{2, t}=\left(I-G_{t} K_{t}\right)^{-1} G_{t} K_{t} D_{t} L_{t}=T_{1, t} K_{t} D_{t} L_{t}$. For analysis, we define $D_{t} L_{t}=\bar{L}_{t} \bar{M}_{t}$, where

$$
\bar{L}_{t}=\left[\begin{array}{l|llll}
\mathbf{0} & 2^{-\sum_{i=0}^{t} R_{0 i}} & & & \\
& & 2^{-\sum_{i=1}^{t} R_{1 i}} & & \\
& & \ddots & \\
& & & 2^{-R_{t t}}
\end{array}\right],
$$

is a $(t+1) \times \frac{(t+1) t}{2}$ matrix, and

$$
\bar{M}_{t}=\left[\begin{array}{c|c}
\mathbf{0} & \mathbf{0} \\
\hline \mathbf{0} & M_{t}
\end{array}\right],
$$

is a $\frac{(t+1) t}{2} \times \frac{(t+1) t}{2}$ matrix.

The following theorem then gives sufficient conditions for input-output stability. The proof can be found in [9]. Note that for an infinite-dimensional matrix $A$, its corresponding finite matrix $A_{t}$ is the first $(t+1) \times(t+1)$ block of $A$. Also, note that for a matrix $A,\|A\|_{1}=\max _{i} \sum_{j}\left|a_{i j}\right|$. 
Theorem 3.1 Given $r \in l_{\infty, \bar{r}}$, and a rate matrix $\mathcal{R}$, if $\left\|T_{1}\right\|_{1}<\infty$, and $\left\|T_{1} K \bar{L}\right\|_{1}<1$, then there exists a constant scale matrix $M=m I$, such that

1. (stability) $\|y\|_{\infty} \leq\left\|T_{1}\right\|_{1}\|r\|_{\infty}+m\left\|T_{1} K \bar{L}\right\|_{1}$,

2. (quantizer validity) $\|y\|_{\infty} \leq m$.

The last inequality comes from our choice of $m .^{2}$

\section{DECAYING REFERENCE INPUTS}

We now consider the case where $r \in \mathcal{C}_{\gamma, \bar{r}}$, and derive sufficient conditions for input-output stability. We show that if $G$ and $K$ are finite-dimensional systems, the conditions can guarantee that the output signal, $y(t)$, decays exponentially over time. Throughout this section, $G$ and $K$ are assumed to be finite-dimensional systems.

With perfect feedback and a stabilizing $K$, exponentially decaying reference signals generate system outputs that exponentially decay over time. We would like to generate the same types of decaying responses with limited-rate feedback, and thus consider the quantizer scales, $M_{t t}=$ $m \beta^{t}$, to be a decaying function of $t$, where $m<\infty$ and $0 \leq \beta<1$. The matrix $L_{t}$ will then have diagonal elements $l_{k s}=m \beta^{k} 2^{-\sum_{i=k}^{s} R_{k i}}$ for $s=0,1, \ldots, t$ and $k=$ $0,1, \ldots, s$, and the quantizer is valid only if $|y(t)| \leq m \beta^{t}$ for all $t \geq 0$. We assume the following norm conditions,

$$
\begin{aligned}
& \text { 1. }\left\|T_{1}\right\|_{1}<\infty, \\
& \text { 2. }\left\|T_{1} K\right\|_{1}<\infty,
\end{aligned}
$$

and break down $y(t)$ as follows:

$$
y(t)=\left(T_{1} r\right)(t)+\left(T_{1} K D L w_{a}\right)(t)=y_{r}(t)+y_{q}(t) .
$$

Under the first assumption, we show that there exists a positive finite constant $C_{1}$ and an $\alpha_{1}\left(0 \leq \alpha_{1}<1\right)$, such that $\left|y_{r}(t)\right| \leq C_{1} \alpha_{1}^{t}$, for all $t \geq 0$.

Assuming that $\left\|T_{1}\right\|_{1}<\infty$, there exists a $\rho$, with $0 \leq$ $\rho<1$, and a positive finite constant $\eta_{1}$, such that $\left|T_{1, i}\right| \leq$ $\eta_{1} \rho^{i}$, for all $i \geq 0 . T_{1, i}$ is the $i$ th value of the impulse response of $T_{1}$. In fact, $\rho$ can be chosen to be the spectral radius of the stable $A$ matrix of a state-space description for $T_{1}$. Since $T_{1}$ is LTI, $y_{r}(t)=\sum_{i=0}^{t} T_{1, t-i} r_{i}$, therefore,

$$
\begin{gathered}
\left|y_{r}(t)\right| \leq \sum_{i=0}^{t}\left|T_{1, t-i}\right|\left|r_{i}\right| \\
\leq \bar{r} \sum_{i=0}^{\infty} \eta_{1} \rho^{t-i} \gamma^{i} \\
=\eta_{1} \bar{r} \rho^{t} \sum_{i=0}^{\infty}\left(\frac{\gamma}{\rho}\right)^{i} \\
=\frac{\eta_{1} \bar{r} \rho}{\rho-\gamma} \rho^{t},
\end{gathered}
$$

where the last equality holds if $\gamma<\rho<1$. Similarly, $\left|y_{r}(t)\right|=\left|\sum_{i=0}^{t} T_{1, i} r_{t-i}\right| \leq \frac{\eta_{1} \bar{r} \gamma}{\gamma-\rho} \gamma^{t}$, if $\rho<\gamma<1$. Putting both cases together, we get that $\left|y_{r}(t)\right| \leq C_{1} \alpha_{1}^{t}$, for all $t \geq 0$, where

$$
C_{1}=\max \left(\frac{\eta_{1} \bar{r} \gamma}{\gamma-\rho}, \frac{\eta_{1} \bar{r} \rho}{\rho-\gamma}\right), \quad \alpha_{1}=\max (\rho, \gamma) .
$$

\footnotetext{
${ }^{2}$ The stability condition in Theorem 3.1 is sufficient as we have not yet proven that $|y(k)|>m$, for any $k \geq 0$, renders the system unstable
}

Under the second assumption, $\left\|T_{1} K\right\|_{1}<\infty$, we show that there exists a positive finite constant $C_{2}$ and a constant $\alpha_{2}\left(0 \leq \alpha_{2}<1\right)$, such that $\left|y_{q}(t)\right| \leq C_{2} \alpha_{2}^{t}$, for all $t \geq 0$.

Since $\left\|T_{1} K\right\|_{1}<\infty$, there exists a constant $\nu$, with $0 \leq$ $\nu<1$, and a positive constant $\eta_{2}$, such that $\left|\left\{T_{1} K\right\}_{i}\right| \leq$ $\eta_{2} \nu^{i}$, for all $i \geq 0$. We now look at the magnitude of the response due to the exogenous disturbance induced by the quantizer, $y_{q}(t)$.

$$
\begin{gathered}
\left|y_{q}(t)\right|=\left|\sum_{i=0}^{t}\left\{T_{1} K\right\}_{t-i}\left(m \beta^{i} 2^{-\sum_{l=0}^{t} R_{i l}}\right)\right| \\
\leq\|\bar{L}\|_{1} \sum_{i=0}^{\infty}\left|\left\{T_{1} K\right\}_{t-i}\right|\left(m \beta^{i}\right) \\
\leq\|\bar{L}\|_{1} \eta_{2} m \sum_{i=0}^{\infty} \nu^{t-i} \beta^{i} \\
=\|\bar{L}\|_{1} \eta_{2} m \nu^{t} \sum_{i=0}^{\infty}\left(\frac{\beta}{\nu}\right)^{i} \\
=\frac{\|\bar{L}\|_{1} \eta_{2} m \nu}{\nu-\beta} \nu^{t}
\end{gathered}
$$

where the last equality holds if $\beta<\nu<1$, and $\bar{L}$ is defined as in section 3. If $\nu<\beta<1$, then it is easy to show that $\left|y_{q}(t)\right| \leq \frac{\| \bar{L}||_{1} \eta_{2} m \beta}{\beta-\nu} \beta^{t}$. We then get that $\left|y_{q}(t)\right| \leq C_{2} \alpha_{2}^{t}$, for all $t \geq 0$, where

$$
C_{2}=\max \left(\frac{\|\bar{L}\|_{1} m \eta_{2} \beta}{\beta-\nu}, \frac{\|\bar{L}\| \|_{1} m \eta_{2} \nu}{\nu-\beta}\right), \quad \alpha_{2}=\max (\nu, \beta) .
$$

Recall that $\beta$ is a parameter we are looking for to ensure that $|y(t)| \leq m \beta^{t}$. Therefore, $\beta \geq \nu$, which gives us

$$
C_{2}=\frac{\|\bar{L}\|_{1} m \eta_{2} \beta}{\beta-\nu}, \quad \alpha_{2}=\beta .
$$

Putting everything together, we get that for all $t \geq 0$,

$$
|y(t)| \leq\left|y_{r}(t)\right|+\left|y_{q}(t)\right| \leq C_{1} \alpha_{1}^{t}+\frac{\|\bar{L}\|_{1} m \eta_{2} \beta}{\beta-\nu} \beta^{t} .
$$

For the quantizer to be valid, we require $|y(t)| \leq m \beta^{t}$, for all $t \geq 0$. The above observations lead us to the following theorem.

Theorem 4.1 Assume that $T_{1}$ and $K$ are finite-dimensional stable LTI systems, whose corresponding A matrices (of state-space descriptions) have spectral radii $\rho$ and $\nu$, respectively (both $\rho$ and $\nu$ have magnitudes less than 1). Given $r \in \mathcal{C}_{\gamma, \bar{r}}$, and a rate matrix $\mathcal{R}$, if

$$
\begin{aligned}
& \text { 1. }\left|\left\{T_{1}\right\}_{i}\right| \leq \eta_{1} \rho^{i} \quad\left(0 \leq \eta_{1}<\infty\right) \text { for all } i \geq 0, \\
& \text { 2. }\left|\left\{T_{1} K\right\}_{i}\right| \leq \eta_{2} \nu^{i} \quad\left(0 \leq \eta_{2}<\frac{1}{\|\bar{L}\|_{1}}\right) \text { for all } i \geq 0, \\
& \text { 3. } \max (\nu, \rho, \gamma)\left\{1-\eta_{2}\|\bar{L}\|_{1}\right\}>\nu,
\end{aligned}
$$

then there exists a decaying scale matrix $M\left(M_{k k}=m \beta^{k}\right)$ such that

- (stability) $\|y\|_{\infty} \leq\left\|T_{1}\right\|_{1}\|r\|_{\infty}+m\left\|T_{1} K\right\|_{1}$,

- (quantizer validity) $|y(t)| \leq m \beta^{t} \forall t \geq 0$.

\section{Proof:}

Define $C_{1}=\max \left(\frac{\eta_{1} \bar{\gamma} \gamma}{\gamma-\rho}, \frac{\eta_{1} \bar{r} \rho}{\rho-\gamma}\right)$, as discussed in section 4 above. Then choose $\beta=\max (\rho, \nu, \gamma)$ and $m=$ $\frac{C 1}{1-\frac{\eta_{2} \beta\|\bar{L}\|_{1}}{\beta-\nu}}$. It is straightforward to show that $m$ is finite and 
positive due to condition (3). To show input-output stability, we have

$$
\begin{aligned}
\left\|\mathbf{y}^{t}\right\|_{\infty} & =\sup _{t}\left\{\left\|T_{1, t} \mathbf{r}^{t}+T_{2}(t) \mathbf{w}_{a}^{t}\right\|_{\infty}\right\} \\
& \leq\left\|T_{1}\right\|_{1}\|r\|_{\infty}+m\left\|T_{1} K \bar{L}\right\|_{1} \\
& \leq\left\|T_{1}\right\|_{1}\|r\|_{\infty}+m\left\|T_{1} K\right\|_{1} .
\end{aligned}
$$

where the last inequality comes from the fact that $\|\bar{L}\|_{1} \leq$ 1. To show quantizer validity, we set

$$
|y(t)| \leq C_{1} \alpha_{1}^{t}+\frac{\|\bar{L} \mid\|_{1} m \eta_{2} \beta}{\beta-\nu} \beta^{t} \leq m \beta^{t},
$$

where the last inequality comes from our choices of $\beta$ and $m$.

\section{CHARACTERIZATION OF STABLE RATE MATRICES}

Sufficient conditions for input-output stability for both bounded and exponentially decaying reference inputs require $\left\|T_{1} K \bar{L}\right\|_{1}<1$, (or $\left\|T_{1} K \bar{L}\right\|_{1}$ less than some constant) which can be written as a set convex constraints on the rate matrix components. The following theorem shows this result.

Theorem 5.1 Let $X=\{\operatorname{vec}(\mathcal{R})\}^{3}=\left[\begin{array}{llll}R_{00} & R_{01} & R_{02} & \ldots\end{array}\right.$ $\left.\begin{array}{lll}R_{11} & R_{12} & \ldots\end{array}\right]^{\prime}$, then for any infinite dimensional matrix, $P$, the condition $\|P \bar{L}(X)\|_{1}<\alpha$ is convex in $X$ for any $\alpha>0$.

\section{Proof:}

$$
\|P \bar{L}(X)\|_{1}<\alpha \Leftrightarrow \sum_{j=0}^{\infty} f_{j}(X)\left|P_{i j}\right|<\alpha \quad i=0,1, \ldots
$$

where $f_{j}(X)=2^{-\sum_{i=j}^{\infty} R_{j i}}$. We now show that $f_{j}(X)$ is convex in $X$, and thus any non-negative combination of $f_{j}(X)$ is convex. First, we recall that $2^{-a}$ is a convex function of $a$. Let $2^{-\sum_{i=j}^{\infty} R_{j i}}=2^{-c_{j}^{\prime} X}$, where $c_{j}^{\prime}$ is a row vector for $j=0,1, \ldots$

$$
\begin{aligned}
2^{-\lambda c_{j}^{\prime} X_{1}-(1-\lambda) c_{j}^{\prime} X_{2}} & =2^{-\lambda a_{1}-(1-\lambda) a_{2}} \\
& \leq \lambda 2^{-a_{1}}+(1-\lambda) 2^{-a_{2}} \\
& =\lambda 2^{-c_{j}^{\prime} X_{1}}+(1-\lambda) 2^{-c_{j}^{\prime} X_{2}} .
\end{aligned}
$$

If we let $P=T_{1} K$, then we get that the stability condition, $\left\|T_{1} K \bar{L}\right\|_{1}<\alpha$, for any non-negative constant $\alpha$, is a set of convex constraints on the infinite dimensional vector $\operatorname{vec}(\mathcal{R})$. This result allows the search for the most efficient quantizer to be formulated as a convex optimization problem.

\footnotetext{
${ }^{3}$ The "vec" operator on a matrix simply concatenates all the column to form one large column vector.
}

\section{SYNTHESIS OF BIT-ALLOCATION STRATEGIES}

In this section, we synthesize rate matrices for a class of finite-memory quantizers that minimize the channel rate required for input-output stability.

\subsection{Finite-Memory Quantizers}

In [9], we introduced a special class of practical quantizers that have finite memory and are periodic. Specifically, each value of $y$ gets approximated by the quantizer for $a t$ most $N$ consecutive time steps. In fact, for any $t \geq 0$, $y(t N+j)$ gets approximated for $N-j$ time steps, for $j=0,1, \ldots, N-1$. Moreover, the bit-allocation strategy repeats every $N$ time steps. We call this class of quantizers, "repeated-block" (RB) quantizers because the structure of the rate matrix is block diagonal as shown below.

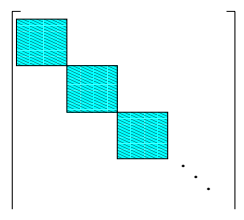

Each block is the following $N \times N$ matrix:

$$
R_{\text {block }}=\left[\begin{array}{cccc}
R_{00} & & & \\
R_{01} & R_{11} & & \\
\vdots & \vdots & \ddots & \\
R_{0, N-1} & R_{1, N-1} & \cdots & R_{N-1, N-1}
\end{array}\right]
$$

RB quantizers are time-invariant operators in "lifted" coordinates, where each time step in the lifted coordinates is equivalent to $N$ time steps in original coordinates.

To find the minimum channel rate required for inputoutput stability and the corresponding bit-allocation strategy, for a given plant and stabilizing controller, we solve the following convex optimization problem,

$$
\begin{aligned}
& \begin{array}{ll} 
& \min R \\
\text { s.t. } & R=\sum_{j} R_{i j} \quad i=0,2, . ., N-1
\end{array} \\
& \begin{array}{c}
\left.\|(I-\tilde{G} \tilde{K})^{-1} \tilde{G} \tilde{K}\right) \tilde{L}_{N} \|_{1}<1, \quad j<i=0,2, \ldots, N-1, \\
R_{i j}>0
\end{array}
\end{aligned}
$$

which is readily computable for the class of finite-memory quantizers with $N \times N$ repeated-block rate matrices.

For a given $G$ and $K$, we denote the state-space description of $(I-\tilde{G} \tilde{K})^{-1} \tilde{G} \tilde{K}$ by $\left(\tilde{A}_{c l}, \tilde{B}_{c l}, \tilde{C}_{c l}, \tilde{D}_{c l}\right)$, and define $X=\operatorname{vec}\left(R_{\text {block }}\right)$ and rewrite the above optimization problem as

$$
\begin{gathered}
\min X(1) \\
\text { s.t. } \quad A_{e q} X=B_{e q}, \\
P(X)<0, \\
X \geq 0,
\end{gathered}
$$


Table 1. Optimal Bit-Allocation Strategies For Various Closed-Loop LTI Systems: $R_{\min }$ denotes the minimum rate required for stability when a memoryless quantizer is in the loop.

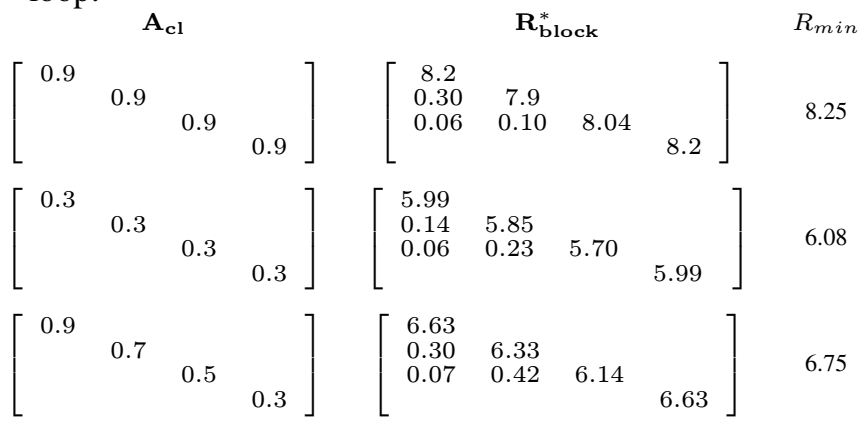

where $A_{e q} X=B_{e q}$ captures the equality constraints (1), $P(X)<0$ captures constraints (2) and $X \geq 0$ are equivalent to constraints (3). We approximate the 1-norm of $(I-\tilde{G} \tilde{K})^{-1} \tilde{G} \tilde{K} \tilde{L}_{N}$, to guarantee that we can numerically compute the solution to the constrained minimization problem.

We now set $N=4$, and solve for the most efficient quantizers that maintain input-output stability for a variety of plant and controller pairs. We denote the state space description $(I-G K)^{-1} G K$ by $\left(A_{c l}, B_{c l}, C_{c l}, D_{c l}\right)$. The statespace description of the lifted system can easily be computed from $\left(A_{c l}, B_{c l}, C_{c l}, D_{c l}\right)$ as described in [12]. To solve for optimal bit-allocation strategies, we used MATLAB's function 'fmincon.m'.

Table 1 shows the resulting optimal $R_{b l o c k}^{*}$ for different closed-loop LTI systems. We allow $A_{c l}$ to vary, and fix $B_{c l}=-\left[\begin{array}{llll}1 & 2 & 1 & 10\end{array}\right]^{\prime}, C_{c l}=\left[\begin{array}{llll}1 & 5 & 3 & 1\end{array}\right]$, and $D_{c l}=0$. We also consider memoryless quantizers $(N=1)$, i.e., $R$ bits are allocated to every input of the quantizer, for the same plants and controllers. In this case, $R_{\min }>$ $\log \left(\left\|T_{1} K\right\|_{1}\right)$. The last column of Table 1 shows the minimum rate required for stability for memoryless quantizers. ble 1 .

There are a few observations one can gleam from Ta-

- The larger the spectral radius of $A_{c l}$, the larger $R_{\min }$ must be for stability. The component of $\tilde{y}$ that decays most slowly forces the channel to have larger rates for stability.

- The optimal strategy tries to allocate more bits to the output components of $\tilde{y}$ that decay at slower rates, i.e., those components that are functions of states that have eigenvalues with larger magnitudes. This is intuitive as the signals that decay more slowly are larger in magnitude over time and hence the quantizer is more likely to generate more errors on these signals if it fails to allocate sufficient bits to them.

- When we compare the minimum rates for the finite-memory RB quantizers and memoryless quantizers, we see that forcing the quantizer to be memoryless requires the channel to have larger transmission rates to maintain input-output stability. Therefore, stability may be achieved for channels with low rates by allowing the quantizer to have more memory.

\section{CONCLUSIONS AND FUTURE WORK}

In summary, we have constructed a parameterization of general time-varying quantizers that leads to a convex characterization of bit-allocation strategies that maintain inputoutput stability for two classes of reference inputs. For finite memory quantizers, the convex characterization of stabilizing quantizers allows for efficient time-varying bit-allocation strategies to be synthesized for a given plant and controller.

Future work includes synthesizing LTI controllers that maintain stability and that achieve specified performance objectives.

\section{REFERENCES}

[1] Baillieul, J., "Feedback Coding for Information-based Control: Operating Near the Data-Rate Limit," Proceedings of the 41st IEEE Conference on Decision and Control, December 2002.

[2] Brockett, Roger W., "Quantized Feedback Stabilization of Linear Systems,'IEEE Transactions on Automatic Control: vol. 45, pp. 1279-1289, 2000.

[3] Wong, Wing Shing, Brockett, Roger, "Systems with Finite Communication Bandwidth Constraints-II: Stabilization with Limited Information Feedback," IEEE Transactions on Automatic Control: vol. 44, no. 5, May 1999.

[4] Delchamps, David, "Stabilizing a Linear System with Quantized State Feedback," IEEE Transactions on Automatic Control: vol. 35, no. 8, August 1990.

[5] Elia, Nicola, Mitter, Sanjoy "Stabilization of Linear Systems With Limited Information," IEEE Transactions on Automatic Control: vol. 46, no. 9, September 2001.

[6] Ishii, Hideaki, Francis, Bruce, "Limited data rate in control systems with networks," Berlin; New York: Springer, c2002.

[7] Liberzon, Daniel, "A note on stabilization of linear systems using coding and limited communication," Proceedings of the 41st IEEE Conference on Decision and Control, December 2002 .

[8] Nair, G.N., Evans, R.J., "Stabilization with Data-rate-limited Feedback: tightest attainable bounds," Systems and Control Letters, volume 41, 2000, pp. 49-56.

[9] Sarma, Sridevi, Salapaka, Srinivasa, Dahleh, Munther A., "A Convex Parameterization of Limited-Rate TimeVarying Quantizers Maintaining Closed-Loop Stability," 42nd Mediterranean Conference on Control and Automation, June 2004.

[10] Tatikonda, Sekhar, "Control Under Communication Constraints," M.I.T. PhD : 2000.

[11] Yuksel, Serder, Basar, Tamer, "State Estimation and Control for Linear Systems over Communication Networks," Proceedings of 2003 IEEE Conference on Control Applications, Volume 1, June 2003.

[12] Chen, Tongwen, Francis, Bruce, "Optimal Sampled-Data Control Systems," Springer-Verlag : Printed in Great Britain, 1996. 